\title{
Examination of Temperature Dependence of Chemical Sputtering on Graphite by Comparing the Langevin and Berendsen Thermostats
}

\author{
Atsushi M. ITO, Hisashi OKUMURA ${ }^{1)}$, Seiki SAITO ${ }^{2)}$ and Hiroaki NAKAMURA ${ }^{2)}$ \\ National Institute for Fusion Science, Oroshi-cho 322-6, Toki 509-5292, Japan \\ ${ }^{1)}$ Research Center for Computational Science, Institute for Molecular Science, \\ 38 Nishigo-Naka, Myodaiji, Okazaki 444-8585, Japan \\ ${ }^{2)}$ Department of Energy Engineering and Science, Graduate school of Engineering, \\ Nagoya University, Furo-cho, Chikusa-ku, Nagoya 464-01, Japan
}

(Received 10 December 2009 / Accepted 22 April 2010)

\begin{abstract}
Chemical sputtering on a graphite $\left(\begin{array}{llll}0 & 0 & 0 & 1\end{array}\right)$ surface due to hydrogen atom bombardment was investigated by a molecular dynamics simulation. To understand the temperature dependence of the sputtering yield of $\mathrm{CH}_{\mathrm{x}}$ and $\mathrm{C}_{2} \mathrm{H}_{\mathrm{x}}$, two thermostat methods used to control material temperature, the Langevin and Berendsen thermostats, were compared. The simulated sputtering yield of $\mathrm{CH}_{4}$ with a peak at $600 \mathrm{~K}$ under the Langevin thermostat agreed with experimental observations. The present simulation showed that the sputtering yield depends on the thermal relaxation time, which is the time required for the temperature to reduce and is the second key parameter for both thermostat methods. In addition, it was demonstrated that the Berendsen thermostat, which controls temperature globally, provides larger sputtering yields of $\mathrm{CH}_{\mathrm{x}}$ and $\mathrm{C}_{2} \mathrm{H}_{\mathrm{y}}$ than the Langevin thermostat, which controls temperature locally.
\end{abstract}

(c) 2010 The Japan Society of Plasma Science and Nuclear Fusion Research

Keywords: molecular dynamics, plasma-wall interaction, chemical sputtering, graphite, carbon

DOI: $10.1585 /$ pfr.5.S2020

\section{Introduction}

In a nuclear fusion device, divertor plates are bombarded by hydrogen plasma. The divertor plates consist of plasma-facing materials (PFM), which are typically carbon, tungsten, and beryllium. Carbon PFM, a low- $Z$ material, contributes to the achievement of high temperatures in core plasma. However, the carbon easily creates chemical bonds with a hydrogen (isotope) atom and ion, resulting in problems such as tritium retention, chemical sputtering, chemical erosion, and hydrocarbon impurity generation and re-deposition. The mechanisms of these chemical processes in carbon PFM are not well understood. A recent trend in research on plasma-wall interaction (PWI) is to evaluate whether carbon or tungsten is more suitable for the divertor plate, especially with regard to tritium retention. The purpose of the present paper is to elucidate the mechanism of chemical sputtering on a graphite surface to evaluate correctly the usefulness of carbon PFM.

To analyze the chemical processes involved, which occur at the atomic scale, PWI between hydrogen plasma and graphite surfaces was solved by a molecular dynamics (MD) simulation. In experimental observations of PWI, hydrocarbons generated by chemical sputtering have often been observed; the dependence of their sputtering yields

author'se-mail: ito.atsushi@nifs.ac.jp on material temperature, incident energy, and flux were discussed. However, it is difficult to reproduce the dependence of sputtering yield on material temperature by MD because MD, which applies at the nanometer and picosecond scale, must set the incident flux of hydrogen atoms to $10^{28}-10^{30}$ atom $/ \mathrm{m}^{2} \mathrm{~s}$, which is much higher than the experimental values of $10^{19}-10^{24}$ atom $/ \mathrm{m}^{2} \mathrm{~s}$. A suitable thermostat method for material temperature in the strong nonequilibrium state caused by the high incident flux of hydrogen atoms has not yet been developed. Though the sputtering yields have been calculated by MD simulations [1,2], their temperature dependence has not yet been presented. We consider that the sputtering yields calculated by MD simulations are affected by not only the setting temperature used in the thermostat method but also the model of the thermostat.

In the present paper, chemical sputtering on a graphite (l $\left.\begin{array}{llll}0 & 0 & 0 & 1\end{array}\right)$ surface bombarded by hydrogen atoms was simulated by $\mathrm{MD}$, and the temperature dependence of the sputtering yields of $\mathrm{CH}_{\mathrm{x}}$ and $\mathrm{C}_{2} \mathrm{H}_{\mathrm{y}}$ using the Langevin and Berendsen thermostats, which are famous thermostat models in general MD, were compared.

\section{Simulation Model}

This section describes the conditions of the MD simulation. A graphite material was first placed on a simula- 
tion box as a layer structure of five graphene sheets stacked in an $\mathrm{ABAB}$ pattern. The graphite material consisted of 800 carbon atoms. The $\left(\begin{array}{llll}0 & 0 & 0 & 1\end{array}\right)$ surface of the material was perpendicular to the $z$ direction and was 20.04 $\times 21.69 \mathrm{~nm}^{2}$ in size. Hydrogen atoms were injected into the surface perpendicularly at random incident positions that were uniformly distributed in the $x$ and $y$ coordinates. During the MD simulation, 1500 hydrogen atoms were injected at $30 \mathrm{eV}$. To maintain the position of graphite material in the simulation box against impacts with hydrogen atoms, six carbon atoms in the deepest graphene sheet were fixed in their initial positions.

Atomic interaction was modeled by two empirical potential functions. One was the modified reactive empirical bond order (REBO) potential, which represents the shortrange forces due to covalent bonds $[3,4]$. The other was the interlayer intermolecular potential with a cone cutoff method, which represents the long-range forces acting between graphene layers [5]. These potential functions were given complicated forms to reproduce the stabilities of several molecular structures. The functions were constructed using not only the distance between atoms but also information about surrounding atoms.

The material temperature was regulated by thermostat methods that act on carbon atoms only. In general, the thermostat methods were characterized by two parameters, the setting temperature $T_{\mathrm{s}}$ and thermal relaxation time $\tau$. In this study, the Langevin and Berendsen [6] thermostats were compared.

In the MD simulation using the Langevin thermostat, the equations of motion of carbon atoms were given by

$$
\dot{p}_{\mathrm{i}}(t)=-\frac{\partial U(r(t))}{\partial r_{\mathrm{i}}}-\gamma p_{\mathrm{i}}(t)+\xi_{\mathrm{i}}(t)
$$

where $r_{\mathrm{i}}$ and $p_{\mathrm{i}}$ are the positions and momenta of atoms, respectively. The coefficient of friction $\gamma$ corresponds to the inverse of the thermal relaxation time as $\tau=1 / 2 \gamma$. Random force $\xi_{\mathrm{i}}(t)$ satisfies

$$
\begin{aligned}
& \left\langle\xi_{\mathrm{i}}(t)\right\rangle=0, \\
& \left\langle\xi_{\mathrm{i}}(t) \xi_{\mathrm{j}}\left(t^{\prime}\right)\right\rangle=2 D \delta\left(t-t^{\prime}\right) \delta_{\mathrm{ij}},
\end{aligned}
$$

where the bracket $\langle\cdots\rangle$ indicates the expected value of random variables, $\delta\left(t-t^{\prime}\right)$ is the Dirac delta function, and $\delta_{\mathrm{ij}}$ is the Kronecker delta. The magnification of random force $D$ is given by the Einstein relation,

$$
D=\gamma m_{\mathrm{i}} k_{\mathrm{B}} T_{\mathrm{s}}
$$

where $m_{\mathrm{i}}=12 \mathrm{u}$ is the mass of a carbon atom and $k_{\mathrm{B}}$ is the Boltzmann constant. In the MD simulation, random force $\xi_{\mathrm{i}}(t)$ was generated by SIMD-oriented Fast Mersenne Twister [7] and the Box-Muller transform [8].

In the MD simulation using the Berendsen thermostat, carbon atoms obey the following equation of motion,

$$
\dot{p}_{\mathrm{i}}(t)=-\frac{\partial U(r(t))}{\partial r_{\mathrm{i}}}+\frac{1}{2 \tau}\left(\frac{T_{\mathrm{s}}}{T(t)}-1\right) p_{\mathrm{i}}(t)
$$

where the momentary temperature $T(t)$ is defined by

$$
T(t)=\frac{2}{3 N k_{\mathrm{B}} \tau_{\mathrm{p}}} \int_{t-\tau_{\mathrm{p}}}^{t} \sum_{\mathrm{i}}^{\text {carbon }} \frac{p_{\mathrm{i}}^{2}\left(t^{\prime}\right)}{2 m_{\mathrm{i}}} \mathrm{d} t^{\prime}
$$

where $N=800$ is the number of carbon atoms.

Under both the Langevin and Berendsen thermostats, $T(t)$ approximately approaches the setting temperature $T_{\mathrm{s}}$ according to an exponential decay curve:

$$
T(t)=\left(T(0)-T_{\mathrm{s}}\right) \exp \left(-\frac{t}{c \tau}\right)+T_{\mathrm{s}} .
$$

The parameter $c=\langle E\rangle /\langle K\rangle$ depends on the potential form, where $E$ and $K$ are the total energy and total kinetic energy, respectively, in a canonical system. If the system is the gas phase, $c \sim 1$, because the total potential energy is about zero; whereas if the system is the solid phase, $c \sim 2$, because the total potential energy is equal to the total kinetic energy by the equipartition of energy. The injection timing of hydrogen atoms was determined as follows. First, we waited for the momentary temperature $T(t)$ to fall into the range that $\left|\langle T(t)\rangle_{\tau_{\mathrm{p}}}-T_{\mathrm{s}}\right|<50 \mathrm{~K}$. Here, the time average of the momentary temperature was calculated for $\tau_{\mathrm{p}}=0.1 \mathrm{ps}$ by

$$
\langle T(t)\rangle_{\tau_{\mathrm{p}}}=\frac{1}{\tau_{\mathrm{p}}} \int_{t-\tau_{\mathrm{p}}}^{t} T\left(t^{\prime}\right) \mathrm{d} t^{\prime} .
$$

This phase continued for at least $0.1 \mathrm{ps}$ and the time step was set to $5 \times 10^{-5} \mathrm{ps}$. Second, one hydrogen atom was injected into the surface normally. The second phase continued for $0.025 \mathrm{ps}$ at a time step of $5 \times 10^{-6} \mathrm{ps}$. The MD simulation subsequently returned to the first waiting phase. These steps were repeated until 1500 hydrogen atoms had been injected.

\section{Results and Discussion}

The simulation results are discussed in terms of the dependences of sputtering yields on the setting temperature $T_{\mathrm{s}}$ and thermal relaxation time $2 \tau$. Here, we define the $\mathrm{CH}_{\mathrm{x}}$ and $\mathrm{C}_{2} \mathrm{H}_{\mathrm{x}}$ yields as the ratio of the numbers of generated $\mathrm{CH}_{\mathrm{x}}$ and $\mathrm{C}_{2} \mathrm{H}_{\mathrm{x}}$ molecules to the number of injected hydrogen atoms, which was 1500. For example, if $15 \mathrm{CH}_{4}$ are generated in the MD simulation, the $\mathrm{CH}_{4}$ yield becomes $10^{-2}$. The MD simulations were performed for setting temperatures $T_{\mathrm{s}}$ of $300,600,900,1200$, and $1500 \mathrm{~K}$ and thermal relaxation times $2 \tau$ of $0.01,0.1,0.33$, and $1.0 \mathrm{ps}$.

Figure 1 shows the $\mathrm{C}$ to $\mathrm{CH}_{4}$ yields. The dependences of the $\mathrm{CHx}$ yields on setting temperature and thermal relaxation time clearly differed between the Langevin and Berendsen thermostats. The result for the Langevin thermostats at $2 \tau=0.33$ ps were comparable to the experimental results. The $\mathrm{CH}_{4}$ yield in this case had a peak value at $600 \mathrm{~K}$, which was $10^{-2}$. This profile of temperature dependence agreed with experimental results [9-17]. In particular, the experimental results in the case of a low incident 


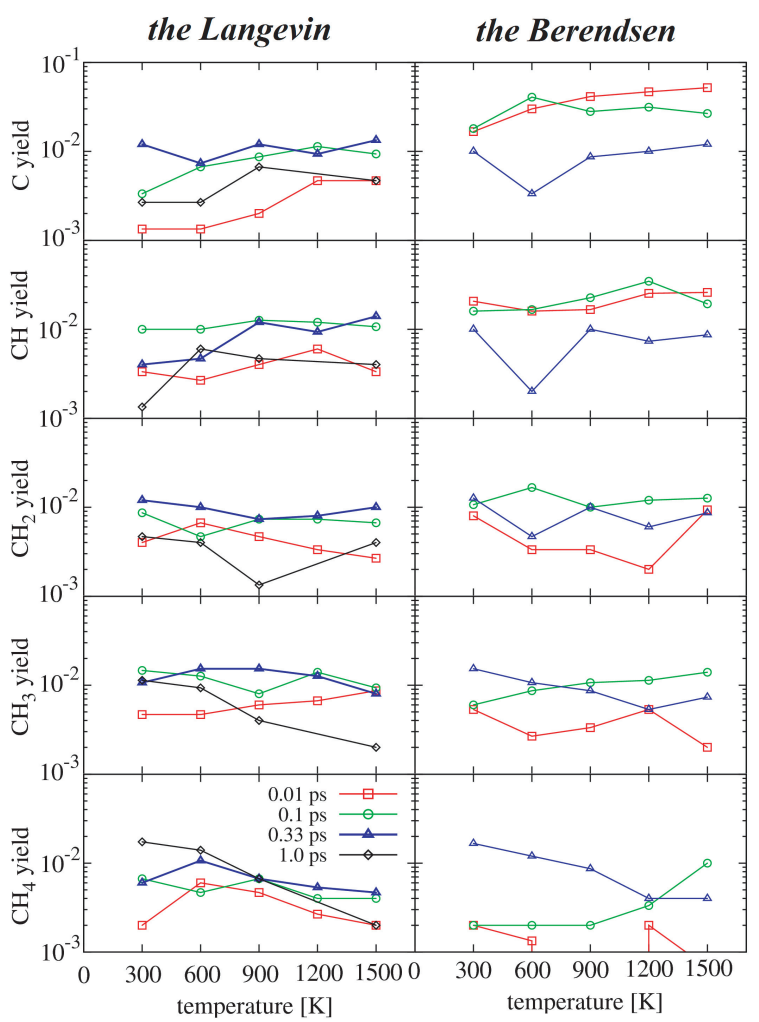

Fig. $1 \mathrm{CH}_{\mathrm{x}}$ yields due to bombardment with 1500 hydrogen atoms for the Langevin and Berendsen thermostats. Printed values of thermal relaxation time correspond to $2 \tau$.

energy of less than $100 \mathrm{eV}$ (for example, $\mathrm{CH}_{4}$ yield due to $\mathrm{H}^{+}$injection at $50 \mathrm{eV}[15]$ ), agreed well. However, for the Berendsen thermostat, the $\mathrm{CH}_{4}$ yield showed no peak. The $\mathrm{CH}_{4}$ yield decreased as the setting temperature increased. However, the absence of a peak at $600 \mathrm{~K}$ was not the only anomalous behavior of $\mathrm{CH}_{4}$ yield. The $\mathrm{C}$ and $\mathrm{CH}$ yields became small at $600 \mathrm{~K}$ and $2 \tau=0.33 \mathrm{ps}$ under both the Langevin and Berendsen thermostats. We consider that the generation rates of $\mathrm{C}$ and $\mathrm{CH}$ were smaller than the growth rates to $\mathrm{CH}_{3}$ and $\mathrm{CH}_{4}$ from $\mathrm{C}$ and $\mathrm{CH}$ via $\mathrm{CH}_{2}$. Therefore, the peak in $\mathrm{CH}_{4}$ yield at $600 \mathrm{~K}$ should be checked by error bar estimation after more simulation sampling.

Both the Langevin and Berendsen thermostats were written with only two parameters, the setting temperature and thermal relaxation time, but they cooled or heated particles by different mechanisms. Cooling and heating by thermostats generally affect the rate of chemical reactions. If the thermal relaxation time is small, the difference in particle motion controlled by thermostats is obvious. We considered that if the parameter was ideal, the difference in $\mathrm{CH}_{\mathrm{x}}$ yields resulting from the difference in thermostats does not appear. The MD simulations in which the thermal relaxation time was 0.1 ps or less, in which the $\mathrm{CH}_{\mathrm{x}}$ yields differed between the two thermostats, could not replicate realistic PWI.

To compare $\mathrm{CH}_{\mathrm{x}}$ and $\mathrm{C}_{2} \mathrm{H}_{\mathrm{y}}$, Fig. 2 shows the total yields of $\mathrm{CH}_{\mathrm{x}}$ and $\mathrm{C}_{2} \mathrm{H}_{\mathrm{y}}$, i.e., the sum of $\mathrm{C}$ to $\mathrm{CH}_{4}$ yields

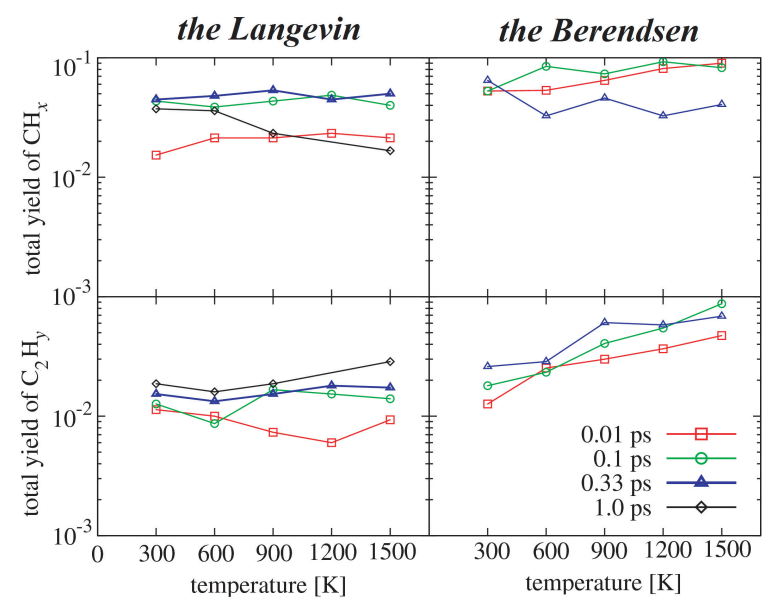

Fig. 2 Total yields of $\mathrm{CH}_{\mathrm{x}}$ and $\mathrm{C}_{2} \mathrm{H}_{\mathrm{y}}$ due to bombardment of 1500 hydrogen atoms under the Langevin and Berendsen thermostats. Printed values of thermal relaxation time correspond to $2 \tau$.

( $x$ was 0-4) and the sum of $\mathrm{C}_{2}$ to $\mathrm{C}_{2} \mathrm{H}_{6}$ yields ( $y$ was $0-6$ ). The dependency of the total yield of $\mathrm{CH}_{\mathrm{x}}$ on setting temperature was weak. For the Langevin thermostat at $2 \tau=$ $1.0 \mathrm{ps}$ only, the total yield of $\mathrm{CH}_{\mathrm{x}}$ decreased as the setting temperature increased. For the Berendsen thermostat, the total yield of $\mathrm{C}_{2} \mathrm{H}_{\mathrm{y}}$ increased as the setting temperature increased. The total yield of $\mathrm{C}_{2} \mathrm{H}_{\mathrm{y}}$ for the Langevin thermostat depended on the setting temperature more weakly than that of the Berendsen thermostat did. In most cases, the total yields of $\mathrm{CH}_{\mathrm{x}}$ and $\mathrm{C}_{2} \mathrm{H}_{\mathrm{y}}$ for the Berendsen thermostat were larger than those of the Langevin thermostat. This difference is considered to be caused by the difference in the temperature control mechanism. The rate of chemical reactions generally depends on the kinetic energy of each atom rather than the temperature of the entire system. According to Eq. (1), the Langevin thermostat maintained the kinetic energy of each atom independent of the other atoms. That is, the Langevin thermostat acts as a local thermostat. Under the Langevin thermostat, local kinetic energy generated by exothermic reactions was absorbed by the thermostat. Because covalent bonds were broken by the local kinetic energy, the detachment of hydrocarbon molecules from surfaces was suppressed under the Langevin thermostat. On the other hand, the Berendsen thermostat checks only the temperature of the entire system by Eq. (5). The Berendsen thermostat did not act on atoms, although the fluctuation in the kinetic energy of atoms was large as long as the momentary temperature $T(t)$ was close to the setting temperature $T_{\mathrm{s}}$. That is, the Berendsen thermostat could be regarded as a global thermostat. The local kinetic energy generated by exothermic reactions was hardly absorbed by the Berendsen thermostat. After the local kinetic energy diffused to surrounding atoms, the entire kinetic energy decreased. Therefore, the covalent bonds were broken more easily under the Berendsen thermostat than under the Langevin thermostat. More- 
over, hydrocarbon molecules were more easily detached from surfaces under the Berendsen thermostat than under the Langevin thermostat. Thus, the fact that the total yields of $\mathrm{CH}_{\mathrm{x}}$ and $\mathrm{C}_{2} \mathrm{H}_{\mathrm{y}}$ for the Berendsen thermostat were larger than for the Langevin thermostat, as shown in Fig. 2, can be explained by this difference between local and global thermostats.

\section{Conclusion Remarks}

The Langevin and Berendsen thermostats were used as a temperature control method to obtain chemical reaction rates and sputtering yields close to experimental results. As a result, a sputtering yield of $\mathrm{CH}_{4}$ with a peak at $600 \mathrm{~K}$, in agreement with experimental results, was observed for the Langevin thermostat at $2 \tau=0.33 \mathrm{ps}$. However, the present MD simulation did not reproduce the chemical sputtering process at low temperatures completely. The sputtering yield of $\mathrm{C}_{2} \mathrm{H}_{\mathrm{y}}$ was greater than that of $\mathrm{CH}_{\mathrm{x}}$ in the MD simulation, whereas the sputtering yield of $\mathrm{C}_{2} \mathrm{H}_{\mathrm{y}}$ was smaller than that of $\mathrm{CH}_{\mathrm{x}}$ in experiments $[14,15]$. In general, the higher the surface temperature is, the larger the hydrocarbon molecules detached from the surface. Therefore, the fact that the sputtering yield of $\mathrm{C}_{2} \mathrm{H}_{\mathrm{y}}$ was greater indicates that the cooling speed of the thermostat was low for the incident flux in the present MD simulation. In future work, the incident flux should be set to a smaller value.

\section{Acknowledgment}

Numerical simulations were performed using the Plasma Simulator at the National Institute for Fusion Science. This study was supported by the National Institutes of Natural Sciences undertaking Forming Bases for Interdisciplinary and International Research through Cooperation Across Fields of Study and by Collaborative Research Program (No. NIFS09KEIN0091), a Grant-in-Aid for JSPS Fellows (No. 20-3829) and a Grant-in-Aid for Scientific Research (No. 19055005) from the Ministry of Education, Culture, Sports, Science and Technology.
[1] E. Salonen, K. Nordlund, J. Keinonen and C. H. Wu, Phys. Rev. B63, 195415 (2001).

[2] E. Salonen, K. Nordlund, J. Keinonen and C. H. Wu, J. Nucl. Mater. 290-293, 144 (2001).

[3] A. Ito, H. Nakamura and A. Takayama, J. Phys. Soc. Jpn. 77, 114602 (2008).

[4] D. W. Brenner, O. A. Shenderova, J. A. Harrison, S. J. Stuart, B. Ni and S. B. Sinnott, J. Phys.: Condens. Matter 14, 783 (2002).

[5] A. Ito and H. Nakamura, Commun. Comput. Phys. 4, 592 (2008).

[6] H. J. C. Berendsen, J. P. M. Postma, W. F. van Gun-steren, A. DiNola and J. R. Haak, J. Chem. Phys. 81, 3684 (1984).

[7] M. Saito and M. Matsumoto, Monte Carlo and QuasiMonte Carlo Methods 2006 (Springer, Berlin Heidelberg, 2008) p. 607.

[8] G. E. P. Box and Mervin E. Muller, The Annals of Mathematical Statistics 29, 610 (1958).

[9] C. H. Skinner, A. A. Haasz, V. KH. Alimov, N Bekris, A. A. Causey, R. E. H. Clark, J. P. Coad, J. W. Davis, R. P. Doerner, M. Mayer, A. Pisarev, J. Roth and T. Tanabe, Fusion Sci. Technol. 54, 891 (2008).

[10] Y. Hirohata, T. Shibahara, T. Tanabe, T. Arai, Y. Gotoh, Y. Oya, H. Yoshida, Y. Morimoto, J. Yagyu, K. Masaki, K. Okuno, T. Hino and N. Miya, J. Nucl. Mater. 337-339, 609 (2005).

[11] J. Roth, R. Preuss, W. Bohmeyer, S. Brezinsek, A. Cambe, E. Casarotto, R. Doerner, E. Gauthier, G. Federici, S. Higashijima, J. Hogan, A. Kallenbach, A. Kirschner, H. Kubo, J. M. Layet, T. Nakano, V. Philipps, A. Pospieszczyk, R. Pugno, R. Ruggieri, B. Schweer, G. Sergienko and M. Stamp, Nucl. Fusion 44, L21 (2004).

[12] A. von Keudell, C. Hopf, T. Schwarz-Selinger and W. Jacob, Nucl. Fusion 39, 1451 (1999).

[13] J. Roth, J. Nucl. Mater. 266-269, 51 (1999).

[14] B. V. Mech, A. A. Haasz and J. W. Davis, J. Nucl. Mater. 255, 153 (1998).

[15] B. V. Mech, A. A. Haasz and J. W. Davis, J. Nucl. Mater. 241-243, 1147 (1997).

[16] J. Roth and C. García-Rosales, Nucl. Fusion 36, 1647 (1996); with corrigendum, Nucl. Fusion 37, 897 (1997).

[17] C. García and J. Roth, J. Nucl. Mater. 196-198, 573 (1992). 